\title{
Primary oral and maxillofacial liposarcoma: a clinicopathological and immunohistochemical study of eleven cases
}

\author{
Jie Cheng ${ }^{1,2}$, Hongbo Yu$u^{1}$, Lizhen Wang ${ }^{3}$, Xudong Wang' ${ }^{1}$ Guofang Shen ${ }^{1}$
}

1Department of Oral and Maxillofacial Surgery, Shanghai Ninth People's Hospital, Shanghai Jiao Tong University, School of Medicine, Shanghai, China

2Department of Oral and Maxillofacial Surgery, Affiliated Stomatological Hospital, Nanjing Medical University, Jiangsu, China

${ }^{3}$ Department of Oral Pathology, Shanghai Ninth People's Hospital, Shanghai Jiao Tong University, School of Medicine, Shanghai, China

Submitted: 10 November 2010

Acccepted: 22 January 2011

Arch Med Sci 2012; 8, 2: 316-323

DOI: 10.5114 /aoms.2012.28560

Copyright @ 2012 Termedia \& Banach

\section{Abstract}

Introduction: The present study was aimed to characterize the clinicopathological, immunohistochemical features and treatment outcomes of primary oral and maxillofacial liposarcomas by presenting the experience over a 16-year period at a tertiary referral Chinese institution for head neck cancer.

Material and methods: This retrospective clinical study included 11 cases of pathologically confirmed primary liposarcomas treated from January 1993 to September 2009. Detailed information regarding primary site, clinical manifestations, histopathological and immunohistochemical analysis, treatments and prognosis was collected and reported.

Results: Eight female and 3 male patients aged from 8 to 76 years old. These lesions occurred in buccal (3), parotid (2), temporal (2), tongue (2), palate (1) and oropharyngeal (1) region. They were histopathologically categorized into 4 subtypes based on WHO classification scheme: atypical lipomatous tumor/welldifferentiated (4), myxoid (4), mixed-type (2) and pleomorphic (1) liposarcomas. Immunohistochemical staining indicated mostly positive for Vimentin and S-100 but negative for other markers. Most patients presented as slow-growing painless masses and underwent surgery alone or combined with postoperative radiotherapy. Two patients were misdiagnosed and inappropriate treated and developed local relapse before referred to our institute. No distant metastasis and one disease-related death were recorded during the follow-up (ranging: 1-11 years, mean: 4.5 years).

Conclusions: Oral and maxillofacial liposarcoma is exceedingly rare and has atypical clinical manifestations but characteristic histopathology. Complete excision with negative margins followed by long-term follow-up is recommended as the treatment of choice for these uncommon entities.

Key words: liposarcoma, oral maxillofacial region, surgery, prognosis.

\section{Introduction}

Liposarcoma represents one of the most common mesenchymal malignant neoplasms, approximately accounting for $20 \%$ of all soft tissue sarcomas [1]. It predominantly occurs in the extremities and retroperitoneum and is extremely rare in the oral and maxillofacial region. To our knowl-

\author{
Corresponding author: \\ Guofang Shen MD, DDS \\ 639 Zhizaoju Road \\ Shanghai 200011, PR China \\ Phone/fax: +86-021-23271699 \\ E-mail: \\ leonardo_cheng@163.com
}


edge, only limited case reports and small series of primary liposarcomas involved in this anatomic region have been previously published [2-7]. The comprehensive clinicopathological information as well as treatment modalities and outcomes of this uncommon malignancy were poorly described thus far. Moreover, the rarity of liposarcoma together with its frequently atypical clinical presentations may probably lead to misdiagnosis and inappropriate management with compromised prognosis. Therefore, a more thorough understanding of the epidemiology, histopathological characteristics of oral and maxillofacial liposarcoma is urgently required to optimize the diagnosis and treatment strategies to achieve better outcomes.

Liposarcomas within oral and maxillofacial region usually present as slow-growing painless mass in individuals with a peak incidence between 40 and 60 years of age [8]. A male preponderance and buccal mucosa as the site of predilection for them were reported $[3,9]$. Advances in genetic and molecular investigation have revealed that liposarcoma is a heterogeneous group of tumors composed of five histological subtypes with distinct morphology, cytogenetic abnormalities and clinical features as shown in the latest WHO classification for soft tissue sarcoma [10]. The precise diagnosis of liposarcoma relies heavily on the histopathological examination before or after surgery. Complete surgical excision remains the mainstream treatment modality for liposarcomas [11]. However, no consensus about the optimized treatment options and proper prognosis prediction for these malignancies exists until now.

In the present study, the clinicopathological and immunohistochemical data, clinical presentation, treatments and disease-specific outcomes regarding primary oral and maxillofacial liposarcomas treated in a tertiary referral Chinese hospital were reported in detail.

\section{Material and methods}

The oral and maxillofacial tumor registry of Shanghai Ninth people's hospital affiliated to Shanghai Jiao Tong University was searched and relevant medical records were reviewed to retrieve information for patients diagnosed as primary oral and maxillofacial liposarcomas between January 1993 and September 2009. Inclusion criteria comprised an oral and maxillofacial location for primary lesion and definitive diagnosis by established pathological criteria for liposarcoma. The archived $H \& E$ and immunohistochemical slides available were further retrieved and reviewed independently by the senior oral pathologists. The research protocol was approved by the Institutional Review Committee of Shanghai Jiao Tong University Medical School. Informed consents were obtained from patients or patient relatives. The clinical and follow-up information of these patients including demographics, primary tumor site, histological subtypes, treatment modality and outcomes from medical records were collected and presented.

During surgery, these lesions were excised about $1.5 \mathrm{~cm}$ away from the tumor margin. After surgical removal of the lesions, the tissue specimen were immediately transferred to oral pathology unit and subjected to pathological examination by frozen sections. The three-dimensional margins were also determined by frozen sections to avoid possible residual tumor. After surgery, the surgical samples were fixed in $10 \%$ buffered formalin and sectioned in 4- $\mu \mathrm{m}$ slides for hematoxylin-eosin staining and immunohistochemical staining using standard procedures. The commercial available antibodies to Vimentin, S-100, CD34, CD99, smooth-muscle actin (SMA), CKpan, Desmin, muscle-specific actin (MSA) were used together with DAB staining system with appropriate positive and negative controls. Immunoreactivity for each protein was determined based on the proportion of stained cells and expected subcellular locations. Negative control was prepared using phosphate buffer solution (PBS) instead of primary and second antibodies.

Most patients in this series underwent complete local excision with disease-free margins. To reduce the possibility of local recurrence, postoperative radiotherapy (about 55-60 Gy) was administered to selected cases. Two recurrent patients who were misdiagnosed and inappropriate treated in local hospital were received another surgical resection in our institute followed by chemotherapy. Large lesions which were more than $5 \mathrm{~cm}$ in largest dimension in other two patients and they were recommended for radiotherapy after surgery. No chemotherapy before and after surgery were given due to the insensitiveness of liposarcoma to chemical agents. All patients were recommended to participate in long-term follow-up at regular intervals.

\section{Results}

\section{Demographic and clinical features}

Through tumor registry search and further pathological confirmation of initial diagnosis, eleven cases of primary oral and maxillofacial liposarcomas were enrolled and briefly showed in Table I. Eight patients were females and the others were males, indicating a female predominance $(8: 3)$. The mean age of patients were 33.7 years. The lesions were found in buccal mucosa (3), temporal (2), parotid (2), ventral tongue (2), palate (1) and the lateral pharyngeal wall (1). Clinical manifestations of this malignancy varied largely depending on the areas involved. Most lesions typically began as slowly-growing and painless masses with dif- 
Table I. Clinicopathological characteristics and treatment outcome of 11 oral and maxillofacial liposarcomas

\begin{tabular}{|lclccccc|}
\hline Case no. & Age [years] & Gender & Primary sites & Pathologic subtype & Treatment & Treatment outcome \\
\hline 1 & 21 & Female & Right parotid region & Myxoid & SE + PR & NED & NED \\
\hline 2 & 26 & Female & Right pharyngeal region & ALT/WD & SE & R, D & NED \\
\hline 3 & 25 & Female & Left buccal region & ALT/WD & SE + PR & SE & NED \\
\hline 4 & 11 & Female & Left buccal region & Pleomorphic & SE & NED \\
\hline 5 & 8 & Female & Left ventral tongue & Myxoid & SE & NED \\
\hline 6 & 46 & Male & Left palate & Mixed-type & SE & NED \\
\hline 8 & 43 & Female & Left temporal region & Myxoid & SE + PR & NED \\
\hline 10 & 28 & Female & Left buccal region & Mixed-type & SE + PR & SE & NED \\
\hline 11 & 48 & Female & Right temporal region & Myxoid & ALT/WD & SE & NED \\
\hline
\end{tabular}

ALT/WD - atypical lipmatous tumor/well differentiated liposarcoma, SE - surgical excision, $S E+P R$ - surgical excision + postoperative radiotherapy, NED - no evidence of disease, $R$-recurrence, $D$ - death

ferent intervals between tumor onset and clinical presentation. The main reasons for patients to seek treatment were accelerated growth of masses and resulting facial disfigurement and the development of pain. Liposarcoma in the parotid region often resembled a begin tumor without obvious facial nerve paralysis. However, when large parotid lesions extended toward parapharygeal space or parapharygeal liposarcoma developed, diverse severities of airway obstruction, dysphagia and night snoring occurred (Figure 1). Computed tomography (CT) and Magnetic resonance imaging (MRI) were usually employed to determine the precise location and boundary as well as its relationship with adjacent tissues. Images of ALT/WD liposarcoma usually showed similar signal intensity equal to fat that closely resemble lipoma and characteristic thickened septa (Figure 1). Other subtypes of liposarcomas showed no remarkable difference to other soft-tissue malignancies in CT/MRI images. Among these patients, wide local excision with negative margins was the most common treatment modality. Radical resection was carried out for patients with local recurrence. Postoperative radiotherapy was also administered to four patients, mainly due to large lesions and recurrent cases. Follow-up period ranged from 1 to 11 years (mean: 4.5 years). Ten patients remained alive until last follow-up, while only 1 patient died of recurrent lesions adjacent to brain and cranial base. Two patients had recurrent lesions about 2-3 months after initial incomplete excision in local hospital before admitted to our department. No evidence of distant metastasis was recorded.

\section{Histopathological and immunohistochemical findings}

Macroscopically, liposarcomas often appeared as soft-tissue mass containing diverse extent of hemorrhage and necrosis and varied from well-circumscribed, multilubolated and partially encapsulated to less well-delineated and more invasive and infiltrating with adjacent tissue. The colors of tumor cut surfaces ranged from yellow to grey and from greasy to mucoid and gelatinous mainly depending on different histopathologic types. Based on histopathology and the latest WHO classification for liposarcoma, eight cases were categorized as atypical lipomatous tumor/well differentiated (ALT/WD) and myxoid liposarcoma. Two cases were subtyped as mixted-type and 1 patient had pleomorphic liposarcoma.

Under microscopy, ALT/WD subtype was characterized by the presence of mature adipocytes with significant variation in cell size and scattered nuclear atypia and variable number of atypical stromal cells (Figure 2). The atypical stromal cells were spindle and often identified with hyperchromatic nuclei embedded within mature adipose tissue. Rare scattered and monovacuolated or multivacuolated lipoblasts could be found. The case with multiple recurrences was diagnosed as lipoma-like subtype of ALT/WD liposarcoma. No evidence of dedifferentiation of ALT/WD or other types of malignancy could be detected in the relapsed lesions. Myxoid liposarcoma consisted round primitive nonlipogenic mesenchymal cells and a few small signet-ring lipoblasts within prominent myxoid stroma (Figure 3). Rich and delicate branching capillary architecture was frequently observed and sometimes vessel and perineural invasions could be detected. As to pleomorphic liposarcoma, it comprised of pleomorphic lipoblasts in the background of plemorphic sarcoma. The plemorphic round and spindled tumor cells were mixed with multinucleated giant cells which were morphologically similar to malignant 

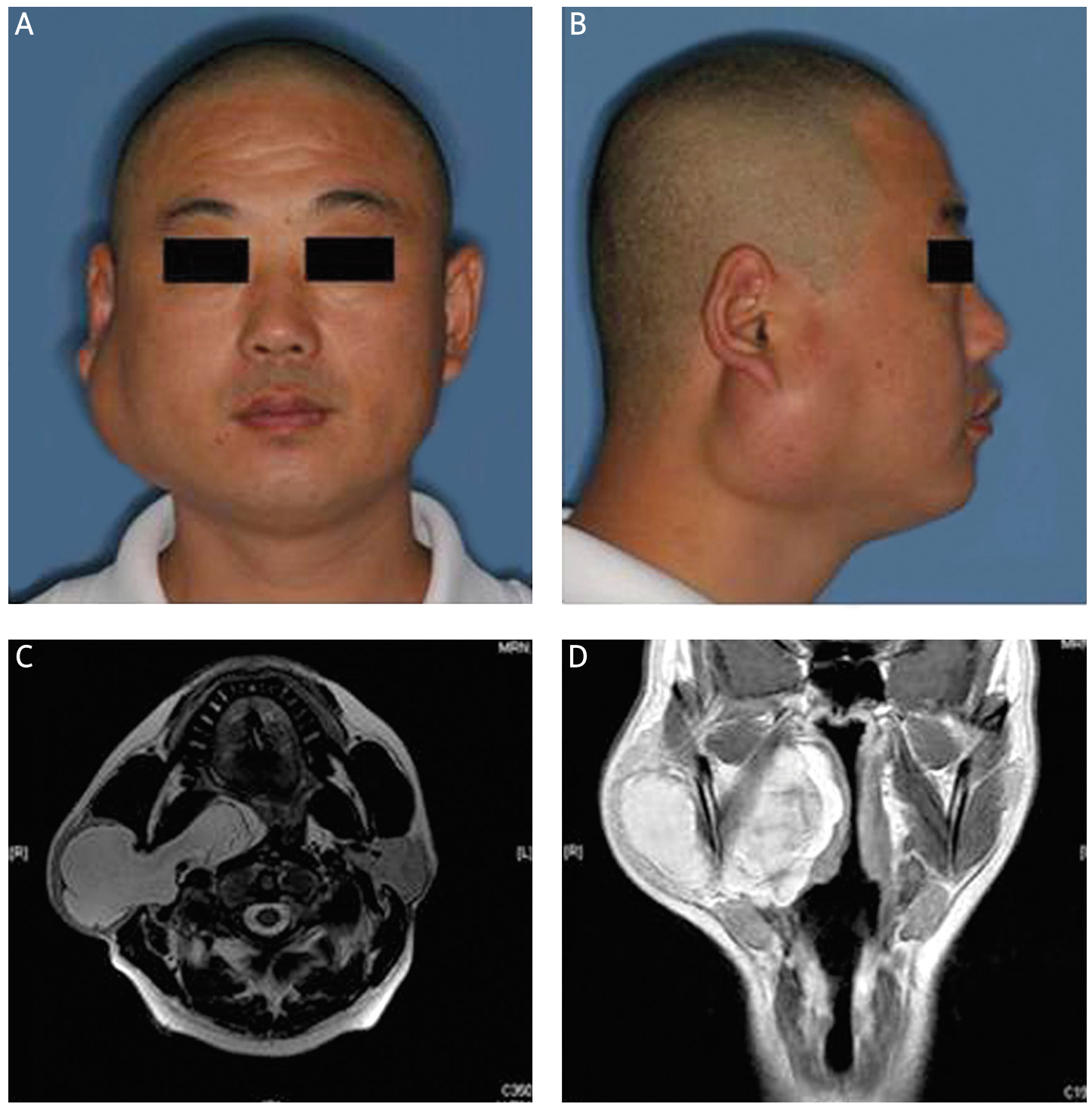

Figure 1. ALT/WD liposarcoma in the right parotid region. A-B - Frontal and lateral view of a male patient with parotid liposarcoma. C-D - MRI images showed a well-defined mass with irregular margins in the right parotid region and extending toward parapharyngeal space ( $C$ - axial fat-suppressed $T 2$ weighted, $D-T 2$ weighted)

fibrous histocytoma (Figure 4). High mitotic activity and intramuscular invasion of tumor could be observed. Mixed-type liposarcoma showed the diverse morphological features of myxoid liposarcoma and ALT/WD or pleomorphic liposarcoma in the lesions with clear boundary. Among the mixedtype lesions, different parts had their own characteristic cell morphology and histopathological features (Figure 5).

Immunohistochemical staining results available for 7 cases showed that vimentin and S-100 were positive most cases. However, CD99, $\alpha$-SMA, MSA, CKp and Des commonly appeared negative (Table II). Despite limited values for direct diagnosis for liposarcoma based on immunohistochemical staining, these results were valuable for differential diagnosis among diverse types of malignancies.

\section{Discussion}

Liposarcoma, first described by Virchow in 1857, is considered as one of the most common soft tissue sarcomas in adults. However, it rarely involves the oral and maxillofacial region $[9,12]$. Its rarity and lack of characteristic symptoms and signs reduced the opportunity to early detect and management [13]. The lack of adequate information regarding the clinicopathological features and prognostic factors for this rare tumor further makes early diagnosis and treatment planning difficult. Our data reported here may improve our understanding of clinicopathological features of oral and maxillofacial liposarcoma to avoid misdiagnosis and mistreatment in the clinic.

Previous reports showed a male/female ratio of about $2: 1$ for liposarcoma in the head and neck 

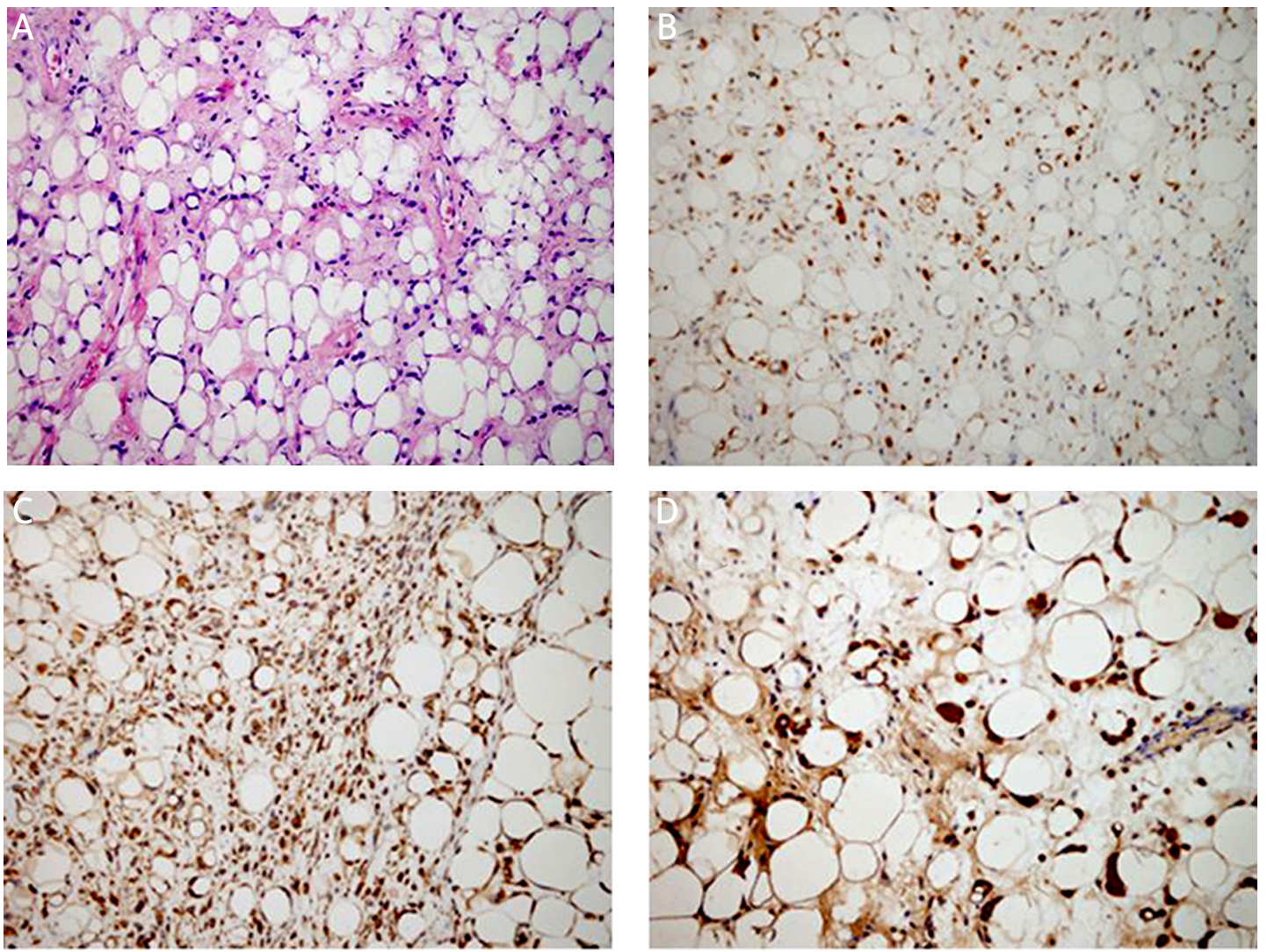

Figure 2. Histopathological and immunohistochemical examination of ALT/WD liposarcoma. Mature adipocytes with diverse size, multi-vacuolated lipoblasts and variable number of atypical stromal cells as showed by $\mathrm{H}+\mathrm{E}$ staining (A). Immunohistochemical staining for vimentin (B), S-100 (C), CD99 (D). Magnification 200x

region, indicating a male preponderance for this tumor $[2,11]$. However, an obvious female predominance (female/male: $8: 3$ ) was found in our series. Similarly, Zheng et al. reported female preponderance with the female/male ratio of $4: 1$ in their series of Chinese origin [5]. The discrepancy of gender distribution may be due to limited number of cases analyzed and/or genetic in origin. Moreover, the average age (33.7 years) in the Chinese cohort is also lower than that in previous studies, as previous reports indicated the age of presentation ranging from 30 and 60 years with a peak in the sixth decades [14]. Notably, two female paediatric patients with 8 and 11 years old were included, being myxoid and pleomorphic subtype arising from buccal mucosa and ventral tongue, respectively. Pediatric liposarcoma is extremely unusual and significantly different from adult cases. Alaggio et al. reported that conventional myxoid liposarcoma was the most common subtype with excellent prognosis in patients younger than 22 year of age [15]. These two paediatric patients underwent wide local excision with negative margins and had no local recurrence and metastasis during the follow-up. This finding reminds the clinicians that oral and maxillofacial liposarcoma can even occur in children and adolescent.
As the sites of predilection for oral liposarcomas, the buccal mucosa is the most common site in this series which is consistent with previous studies [16]. Of note, two parotid liposarcomas were found and both were pathologically classified as ALT/WD. No such lesions were found in minor salivary glands. One patient was initially misdiagnosed as pleomorphic adenoma and inappropriately treated in local hospitals, then developed recurrent lesion 2 month later. To our knowledge, although ten cases of liposarcomas in the parotid region were reported in the English literature so far, only one case with definitive diagnosis of parotid ALT/WD was previously documented by Fanburg-Smith et al. with unspecified treatment and no recurrence during 2 years follow-up [9]. Therefore, our two cases might add some important information to better understanding of parotid ALT/WD and remind surgeons to be alert about the diagnosis and treatment of such rare tumor in the parotid region.

The liposarcomas within oral and maxillofacial region often appear as painless, slow-growing swelling or soft-tissue masses. On the first examination, these disease entities tend to be perceived as benign tumors. Three patients in this series had initial misdiagnosis and incomplete excision and 2 patients developed local recurrence before 

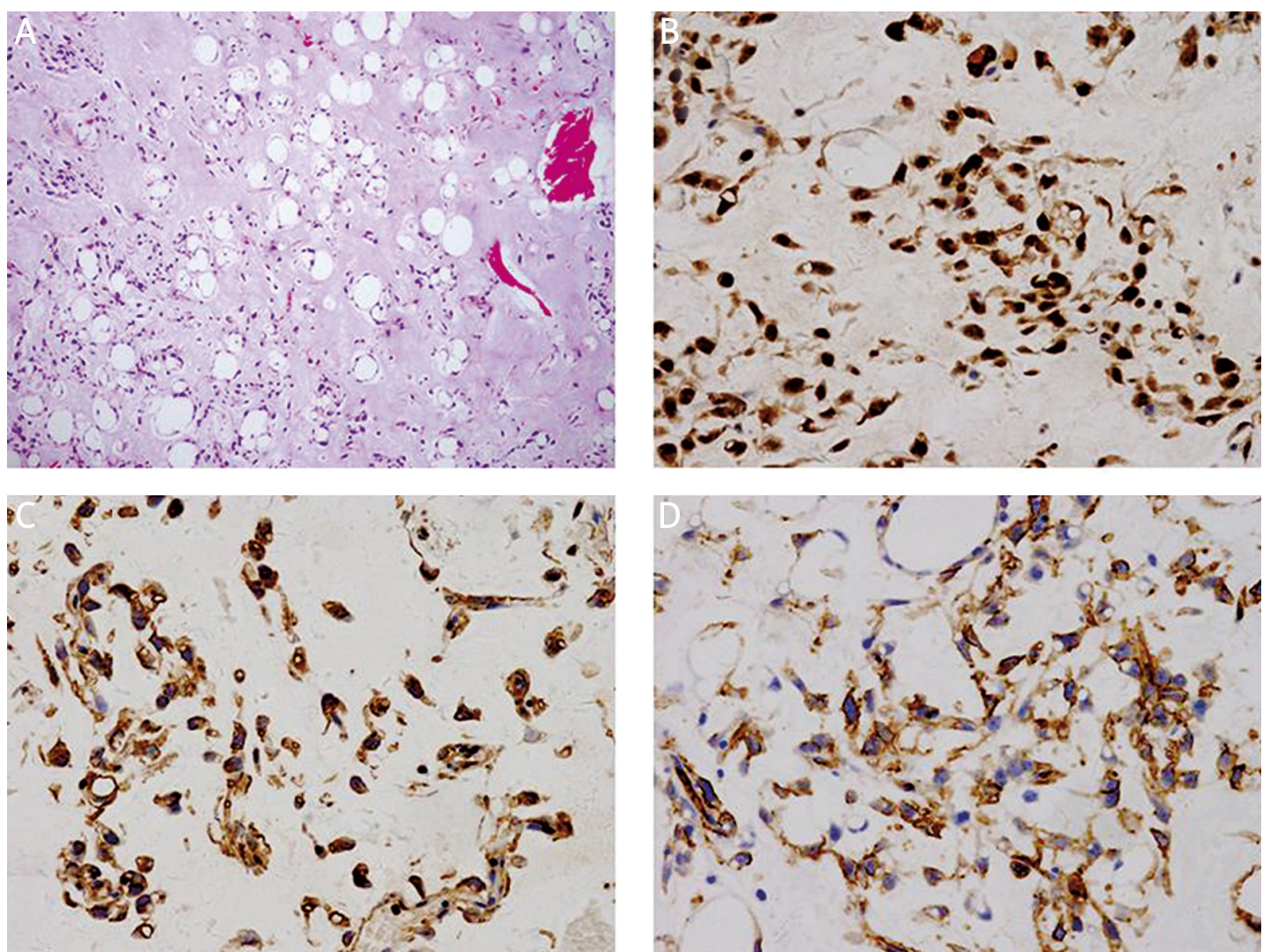

Figure 3. Histopathological and immunohistochemical examination of myxoid liposarcoma. Multivacuolated and signet-ring primitive cells within the prominent myxoid stroma as showed by H\&E staining (A). Magnification 100x. Immunohistochemical staining for, S-100 (B), vimentin (C) CD34 (D). Magnification 400x
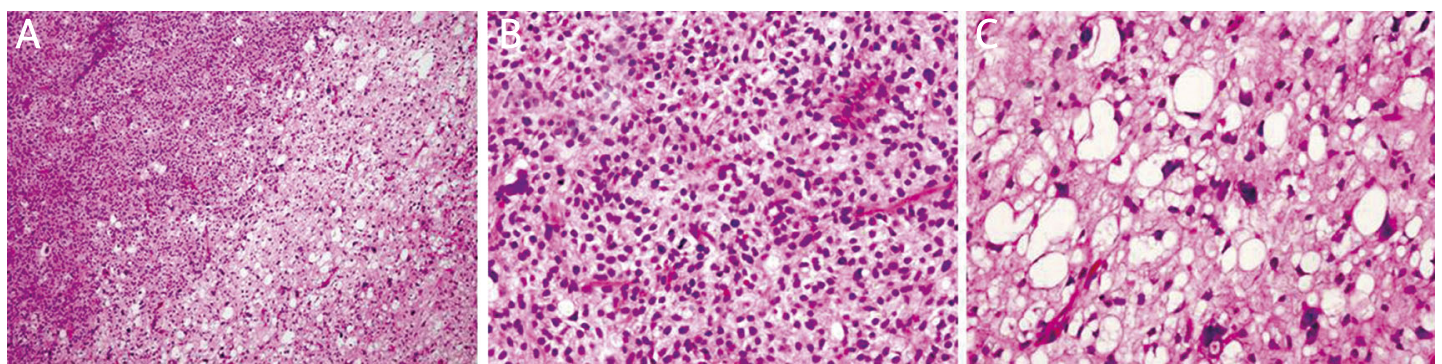

Figure 4. Histopathological examination of pleomorphic liposarcoma. The tumor consists of pleomorphic sarcoma (left area) and pleomorphic lipoblasts (right area) as showed by H\&E staining. Magnification 100× (A). The pleomorphic sarcoma is similar to malignant fibrous histocytoma and contains round and pleomorphic tumor cells with mitosis. Magnification 400× (B). The lipoblasts shown nuclear atypia. Magnification 400× (C)
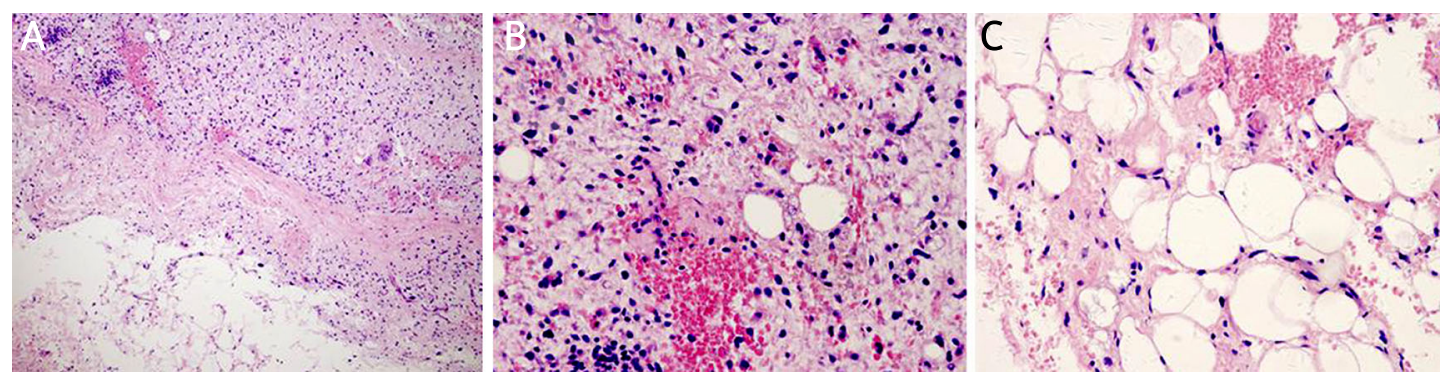

Figure 5. Histopathological examination of mixed-type (ALT/WD \& myxoid) liposarcoma. The lesion consists of ALT/WD (upper area) and myxoid liposarcoma (lower area) with apparent boundary as showed by H\&E staining. Magnification 100x (A). The ALT/WD (B) and myxoid part (C) each shows characteristic histology. Magnification 400x 
Table II. Immunohistochemical analyses of oral and maxillofacial liposarcomas

\begin{tabular}{|lcccccccc|}
\hline Case & Vim & S-100 & CD34 & CD99 & $\alpha-S M A$ & CKp & EMA & Des \\
\hline 1 & + & + & + & - & - & - & - & - \\
\hline 4 & + & - & - & - & - & - & - & - \\
\hline 6 & + & \pm & - & + & - & - & - & - \\
\hline 8 & + & \pm & + & - & - & - & - & - \\
\hline 9 & + & + & - & + & - & - & - & - \\
\hline 10 & + & + & - & \pm & - & - & - \\
\hline 11 & + & + & + & - & - & - & - & - \\
\hline
\end{tabular}

Vim - vimentin, $\alpha$-SMA - $\alpha$-smooth muscle actin, MSA - muscle-specific actin, CKp - pan-cytokeratin, EMA - epithelial membrane antigen, Des-desmin, positive: + , negative: - , partial positive: \pm

referred to our department. Therefore, differential diagnosis of such masses is essential and should include various benign and malignant lesions such as lipomas, lymphadenopathies, branchial cyst and Hodgkin lymphoma, et al. The presence of fat in CT and MRI images may suggest a lipomatous tumor such as lipoma and liposarcoma. Several characteristic findings including more than $75 \%$ fat, thick septa may contribute a lot to differential diagnosis between lipoma and ALT/WD $[17,18]$. However, it's fairly difficult to definitely diagnose such masses between liposarcomas and other soft tissue neoplasm based on radiography.

Histological examination of liposarcoma is pivotal for diagnosis establishment. Tumor cells with characteristic morphology contribute to diagnosis and subtype classifications. The most common histologic subtypes in our series, in agreement with previous findings, are ALT/WD and myxoid liposarcoma. The lipogenic differentiation marker S-100, indicative of the presence of lipoblasts, was stained positive in ALT/WD and myxoid liposarcoma, but negative in other subtypes. Although no specific molecular markers exist for its diagnosis, however, such markers by immunohistochemistry may be beneficial for differential diagnosis and subtype classification. The cytogenetic feature of ALT/WD and dedifferentiated liposarcoma has been revealed in the form of supernumerary ring or giant marker chromosomes with 12q13-15 amplification [19]. Several tumor-associated genes including MDM2, HMGA2 and CDK4 were identified and proven beneficial for differential diagnosis by immunohistochemical detection or fluorescent in situ hybridization [20]. Moreover, fine needle aspiration biopsy was suggested as a valuable and promising technique for preoperative diagnosis especially in the deep region [21]. Therefore, further studies were warranted to determine the roles of such tumor biomarkers in early detection, pathological categorization and prognostic prediction for liposarcoma [22].

Complete surgical excision with free margins is the primary treatment option for liposarcomas [2,
$9,13]$. The values of adjuvant radiotherapy and chemotherapy for liposarcoma still remain controversial. Radiotherapy alone is occasionally considered as an alternative for selected cases especially for impossible total excision or recurrent tumors. Careful follow-up after complete excision has been recommended for monitoring recurrence and distant metastasis. Local recurrence, usually resulting from incomplete excision becomes a common challenge for successful management of liposarcoma. Although these tumors sometimes appear well encapsulated, wide excision should be emphasized to avoid nay microscopic residual disease. In our cases, excisions were performed at least $1.5 \mathrm{~cm}$ from the palpable margins of lesions to guarantee complete removal when possible. However, the proximity of vital neurovascular structures and ensuing severe morbidities caused by wide excision sometimes limited the extent of excision. Therefore, adjuvant postoperative radiotherapy is sometimes needed for selected cases especially when complete excision was unattainable. It has been suggested that radiotherapy delayed tumor growth and prevented local recurrence [23]. Golledge et al. reported that patients treated with surgery alone had a 5 -year survival rate of $83 \%$ as compared with $63 \%$ for those treated with combined surgery and radiotherapy [2]. Four patients reported here underwent surgery combined postoperative radiotherapy and had satisfactory results without evidence of recurrence. Based on our experience and previous reports, adjuvant radiotherapy should be considered as a valuable treatment alternative for local disease control after incomplete excision or suspicious disease left. In addition, no study has offered conclusive evidence supporting the treatment utility of chemotherapy for liposarcoma.

Although previous studies suggested that tumor size, histological subtype and treatment correlated with biological behaviors and prognosis of liposarcomas, we didn't confidentially draw conclusions about the relationship between prognosis and these clinicopathological variables largely due to limited number of cases. But, in general, oral and maxillo- 
facial liposarcomas had good prognosis as supported by our data and others findings, presumably owing to easy detection and timely therapeutic intervention.

In conclusion, we retrospectively reviewed 11 cases of primary liposarcomas within oral and maxillofacial region and described the clinicopathological features and treatment outcomes in detail. Our study indicated that these rare tumors developed from various anatomic sites, most frequently in the buccal mucosa and usually identified as ALT/WD and myxoid subtypes by histopathology. The treatment outcomes of these patients receiving surgery or together with radiotherapy were favorable with $90.9 \%$ disease-free survival during the follow-up, rare recurrence and no metastasis. It's critically essential for clinicians to keep the possible diagnosis of liposarcoma in mind when diagnosing masses in the oral and maxillofacial region. More retrospective and perspective studies are still needed to establish the optimized diagnosis and treatment regimes for oral and maxillofacial liposarcoma.

\section{Acknowledgments}

These works were financially supported by research Grant 2007BAl 118B04 from National Key Technology R\&D Pillar Program in the $11^{\text {th }}$ 5 year plan of China, National Natural Science Foundation of China (Grant No. 81100737) and Natural Science Foundation of Jiangsu Province (Grant No. BK2011762).

\section{References}

1. Mack TM. Sarcomas and other malignancies of soft tissue, retroperitoneum, peritoneum, pleura, heart, mediastinum, and spleen. Cancer 1995; 75: 211-44.

2. Golledge J, Fisher C, Rhys-Evans PH. Head and neck liposarcoma. Cancer 1995; 76: 1051-8.

3. Nascimento AF, McMenamin ME, Fletcher CD. Liposarcomas/atypical lipomatous tumors of the oral cavity: a clinicopathologic study of 23 cases. Ann Diagn Pathol 2002; 6: 83-93.

4. Titiz A, Yilmaz YF, Ceyhan S, Unal T. Atypical lipomatous tumor in the submental region. J Craniofac Surg 2008; 19: 1558-60.

5. Zheng JW, Wang Y. Liposarcoma in the oral and maxillofacial region: an analysis of 10 consecutive patients. J Oral Maxillofac Surg 1994; 52: 595-8.

6. DeWitt J, Heidelman J, Summerlin DJ, Tomich C. Atypical lipomatous tumors of the oral cavity: a report of 2 cases. J Oral Maxillofac Surg 2008; 66: 366-9.

7. Chandan VS, Fung EK, Woods Cl, de la Roza G. Primary pleomorphic liposarcoma of the parotid gland: a case report and review of the literature. Am J Otolaryngol 2004; 25: 432-7.

8. Dei Tos AP. Liposarcoma: new entities and evolving concepts. Ann Diagn Pathol 2000; 4: 252-66.

9. Fanburg-Smith JC, Furlong MA, Childers EL. Liposarcoma of the oral and salivary gland region: a clinicopathologic study of 18 cases with emphasis on specific sites, morphologic subtypes, and clinical outcome. Mod Pathol 2002; 15: 1020-31

10. Fletcher CD, Unni KK, Mertens F (eds). Adipocytic tumors. In: World Health Organization classification of tumors: pathology and genetics of tumors of soft tissue and bone. IARC Press, Lyon 2002; 35-46.

11. Davis EC, Ballo MT, Luna MA, et al. Liposarcoma of the head and neck: The University of Texas M.D. Anderson Cancer Center experience. Head Neck 2009; 31: 28-36.

12. Weiss SW, Goldblum JR. Liposarcoma. In: Enzinger's and Weiss's soft tissue tumors. Weiss SW, Goldblum JR (eds). Mosby, St. Louis 2001; 641-93.

13. Dalal KM, Antonescu CR, Singer S. Diagnosis and management of lipomatous tumors. J Surg Oncol 2008; 97: 298-313.

14. Stewart MG, Schwartz MR, Alford BR. Atypical and malignant lipomatous lesions of the head and neck. Arch Otolaryngol Head Neck Surg 1994; 120: 1151-5.

15. Alaggio R, Coffin CM, Weiss SW, et al. Liposarcomas in young patients: a study of 82 cases occurring in patients younger than 22 years of age. Am J Surg Pathol 2009; 33: 645-58.

16. Charnock DR, Jett T, Heise G, Taylor R. Liposarcoma arising in the cheek: report of a case and review of the literature. J Oral Maxillofac Surg 1991; 49: 298-300.

17. Munk PL, Lee MJ, Janzen DL, et al. Lipoma and liposarcoma: evaluation using CT and MR imaging. AJR Am J Roentgenol 1997; 169: 589-94.

18. Hosono M, Kobayashi H, Fujimoto R, et al. Septum-like structures in lipoma and liposarcoma: MR imaging and pathologic correlation. Skeletal Radiol 1997; 26: 150-4.

19. Micci F, Teixeira MR, Bjerkehagen B, Heim S. Characterization of supernumerary rings and giant marker chromosomes in well-differentiated lipomatous tumors by a combination of G-banding, CGH, M-FISH, and chromosome- and locus-specific FISH. Cytogenet Genome Res 2002; 97: 13-9.

20. Dei Tos AP, Doglioni C, Piccinin S, et al. Coordinated expression and amplification of the MDM2, CDK4, and $\mathrm{HMGI}-\mathrm{C}$ genes in atypical lipomatous tumours. J Pathol 2000; 190: 531-6.

21. Klijanienko J, Caillaud JM, Lagacé R. Fine-needle aspiration in liposarcoma: cytohistologic correlative study including well-differentiated, myxoid, and pleomorphic variants. Diagn Cytopathol 2004; 30: 307-12.

22. de Vreeze RS, de Jong D, Nederlof PM, et al. Added value of molecular biological analysis in diagnosis and clinical management of liposarcoma: a 30-year single-institution experience. Ann Surg Oncol 2010; 17: 686-93.

23. Eeles RA, Fisher C, A'Hern RP, et al. Head and neck sarcomas: prognostic factors and implications for treatment. Br J Cancer 1993; 68: 201-7. 